\title{
Tratamientos térmicos de los aceros sinterizados obtenidos a partir de polvos prealeados $\mathrm{Fe}-1,5 \% \mathrm{Mo}^{(\bullet)}$
}

\author{
J.M. Torralba $^{(*)}$, N. Candela ${ }^{(*)}$, F. Velasco ${ }^{(*)}$, L.E.G. Cambronero ${ }^{(*)}$ y J.M. Ruiz-Prieto ${ }^{(*)}$
}

Resumen Se estudia la influencia que distintos estados de tratamiento térmico ejercen sobre las propiedades mecánicas y físicas de tres aceros aleados con cobre, níquel y molibdeno sinterizados. Los tratamientos térmicos realizados consisten en temple y revenido a distintas temperaturas. El material de partida utilizado para conseguir la aleación de molibdeno ha sido un polvo prealeado de Fe - 1,5 Mo.

Palabras clave: Polvos prealeados. Tratamientos térmicos.

\section{Heat treatments of sintered steels obtained from $\mathrm{Fe}-1,5 \% \mathrm{Mo}$ prealloyed powders}

\begin{abstract}
The influence of several heat treatments on the mechanical and physical properties of three different sintered $\mathrm{Ni}$-Cu-Mo alloyed steels are evaluated. The heat treatments carried out were quenching and tempering at different temperatures. The raw material studied was a $\mathrm{Fe}-\mathrm{Mo}$ prealloyed powder with an $1.5 \%$ Mo content.
\end{abstract}

Keywords: Prealloyed steels. Heat treatments.

\section{INTRODUCCIÓN}

Los polvos prealeados Fe - 1,5 Mo se desarrollaron para su empleo en la fabricación de piezas de acero sinterizado destinadas a ser tratadas térmicamente, preferentemente templadas y revenidas. Estudiados y caracterizados distintos tipos de aceros basados en este tipo de polvo prealeado (1), se plantea como objetivo del presente trabajo el estudio de la respuesta frente a los tratamientos de temple y revenido de algunas de las composiciones seleccionadas a partir de los resultados obtenidos en otro trabajo anterior (1). En el presente, también se

(•) Trabajo recibido el día 27 de abril de 1994.

(*) Dpto. de Ingeniería de Materiales. E.T.S. de Ingenieros de Minas. Universidad Politécnica de Madrid. c/ Ríos Rosas, 21. 28003-Madrid (España). centra el estudio en la influencia de la composición sobre distintas propiedades mecánicas (resistencia a la tracción, alargamiento y dureza) y físicas (densidad y variación dimensional) para distintos estados de tratamiento térmico.

\section{ANTECEDENTES}

Sanderow (2) recopila algunos resultados sobre aceros sinterizados templados y revenidos, pero que previamente se habían sinterizado en condiciones muy alejadas de las utilizadas en la industria. En el trabajo desarrollado por Lindqvist (3), donde prácticamente se presenta el polvo prealeado Astaloy Mo utilizado en el presente trabajo, se estudia la templabilidad de aceros obtenidos a partir de este prealeado, así como su capacidad de ser nitrurado por plasma. Otros investigadores (4 y 5) han tratado 
térmicamente estos aceros, pero al igual que los resultados recopilados en (2), partiendo de condiciones de sinterización no usuales en la industria. Algunas de las nuevas familias de polvos prealeados recientemente desarrolladas por la empresa sueca Höganäs, a partir del Astaloy Mo (6) se recomiendan para tratamientos térmicos de temple en profundidad, obteniéndose, con enfriamiento al aire, estructuras martensíticas-bainíticas.

\section{PROCESO EXPERIMENTAL}

\subsection{Obtención y tratamientos de los aceros estudiados}

Los aceros utilizados para este estudio se han obtenido mediante procesado pulvimetalúrgico a partir del material de la tabla I, y en las condiciones de fabricación descritas en (1) que se resumen a continuación:

- Mezcla (en mezclador de laboratorio): 10 min a 30 r.p.m.

- Compactación uniaxial: $700 \mathrm{MPa}$.

- Sinterización: $1.120{ }^{\circ} \mathrm{C}$ durante $20 \min \left(\mathrm{N}_{2}-5 \%\right.$ $\mathrm{H}_{2}$ ).

Las adiciones estudiadas, a partir de un polvo prealeado de hierro con 1,5 Mo y de una composición de carbono añadido fijo del $0,7 \%$ en masa, son las siguientes: $3 \% \mathrm{Cu}, 3 \% \mathrm{Ni}$ y $2 \% \mathrm{Cu}+4 \%$ Ni.

Los tratamientos térmicos a que fueron sometidos los aceros estudiados en el presente trabajo, fueron el temple y el revenido. El temple se hizo desde una temperatura de austenización de $950{ }^{\circ} \mathrm{C}$, durante $30 \mathrm{~min}$, y se enfrió en aceite a $50^{\circ} \mathrm{C}$. Posteriormente, se realizó el revenido a diferentes temperaturas: $325,400,475$ y $550{ }^{\circ} \mathrm{C}$. Todos los tratamientos térmicos se llevaron a cabo en atmósfera inerte de argón, para evitar oxidaciones del material.

\subsection{Propiedades evaluadas}

\subsubsection{Propiedades físicas}

Las propiedades físicas determinadas fueron la densidad y la variación dimensional. La densidad se determinó por el método de Arquímedes. Al tratarse de materiales porosos, antes de su inmersión en agua deben sellarse por medio de una laca o pintura. La densidad y la variación dimensional se calculan en la forma descrita en (1).

\subsubsection{Propiedades mecánicas}

Las propiedades mecánicas determinadas en este trabajo fueron la dureza, la resistencia a la tracción y el alargamiento.

La dureza se determinó mediante el método Rockwell, escala A. Con el ensayo de tracción, se determinaron la resistencia a la tracción y el alargamiento.

\subsection{Estudio metalográfico}

Todos los materiales se estudiaron por microscopía óptica convencional y se completó dicho estudio con la determinación de microdurezas.

TABLA I.- Composición química y principales propiedades de los materiales de partida estudiados

TABLE I.-Chemical composition and main properties of raw materials

\begin{tabular}{|l|c|c|c|c|c|c|c|c|l|}
\hline \multirow{2}{*}{ Material } & \multicolumn{4}{|c|}{ Composición química, \% } & $\begin{array}{c}\text { Densidad } \\
\text { aparente, } \\
\mathrm{g} / \mathrm{cm}^{3}\end{array}$ & $\begin{array}{c}\text { Vel. de } \\
\text { flujo, } \\
\mathrm{s} / 50 \mathrm{~g}\end{array}$ & $\begin{array}{c}\text { Tamaño de } \\
\text { partícula, } \\
\mu \mathrm{m}\end{array}$ & Tipo de polvo \\
\cline { 2 - 8 } & $\mathrm{C}$ & $\mathrm{Cu}$ & $\mathrm{Ni}$ & $\mathrm{Mo}$ & $\mathrm{Fe}$ & 3,0 & 25 & $20-180$ & $\begin{array}{l}\text { Atomizado en } \\
\text { agua }\end{array}$ \\
Astaloy Mo & - & - & - & 1,5 & resto & 3,0 & & & \\
Cobre & - & $>99,3$ & - & - & - & $2,2-2,6$ & 37 & $45-76$ & Electrolítico \\
Níquel & - & - & $>99,9$ & - & - & $0,7-2,0$ & 32 & $3-5$ & Carbonilo \\
Carbono & $>98,0$ & - & - & - & - & 0,13 & - & $<53$ & Natural \\
\hline
\end{tabular}




\section{RESULTADOS}

\subsection{Propiedades físicas}

\subsubsection{Densidad}

En la figura 1 se muestran los valores de la densidad después del temple y revenido de todos los materiales estudiados. Puede apreciarse cómo la aleación con $3 \% \mathrm{Ni}$ en estado de temple tiene una densidad algo mayor que en la primera temperatura de revenido $\mathrm{y}$, posteriormente, según se aumenta dicha temperatura, la densidad se va incrementando progresivamente. Para el caso del cobre, la tendencia que se prevé es la inversa, es decir, según aumenta la temperatura de revenido, la densidad va disminuyendo, aunque ligeramente. En el caso de la aleación con $2 \% \mathrm{Cu}$ y $4 \% \mathrm{Ni}$, el efecto es, en la mayoría de los casos, intermedio debido a la mezcla de aleantes.

\subsubsection{Variación dimensional}

La figura 2 muestra los valores de la variación dimensional después del temple y revenido de todos los materiales estudiados. La aleación con níquel propicia la disminución de la variación dimensional al pasar del estado de temple al de revenido y, según aumenta la temperatura de revenido, se aprecia en mayor medida el efecto de contracción producido por el níquel. El cobre responde de igual forma que el níquel frente al revenido, pero, en este caso, la variación dimensional es positiva. En la aleación que contiene los dos elementos, la respuesta al revenido es intermedia a lo observado en los casos anteriores, pero con un acercamiento al comportamiento del níquel.

\subsection{Propiedades mecánicas}

\subsubsection{Resistencia a la tracción}

La figura 3 recoge los valores de la resistencia a la tracción después del temple y revenido de todos los materiales estudiados. Se puede observar cómo en los tres tipos de aleaciones, al pasar del estado de temple al de revenido, disminuye la resistencia a la tracción. En el caso particular de los aceros con níquel, se aprecia un ligero aumento de resistencia a $400{ }^{\circ} \mathrm{C} \mathrm{y}$, posteriormente, una nueva disminución. El cobre propicia, con la temperatura de revenido, una disminución de la resistencia hasta una temperatura de $475^{\circ} \mathrm{C}$, con un aumento de dicha propiedad para una temperatura de revenido de $550^{\circ} \mathrm{C}$. La aleación con cobre y níquel se comporta de manera intermedia a los casos anteriores.

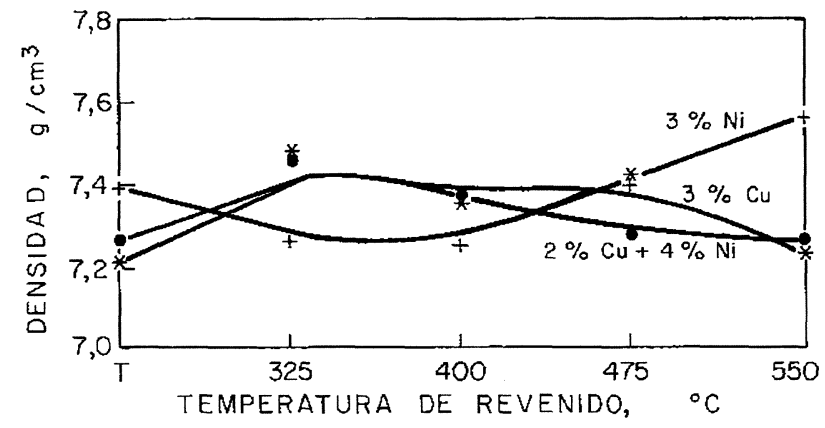

FIG. 1.- Densidad de los aceros Fe-1,5 Mo-0,7 C, templados a $950{ }^{\circ} \mathrm{C}$ y revenidos a distintas temperaturas.

FIG. 1.-Density of Fe-0.7\% C-1.5\% Mo steels, quenched at $950^{\circ} \mathrm{C}$, and tempered at different temperatures.

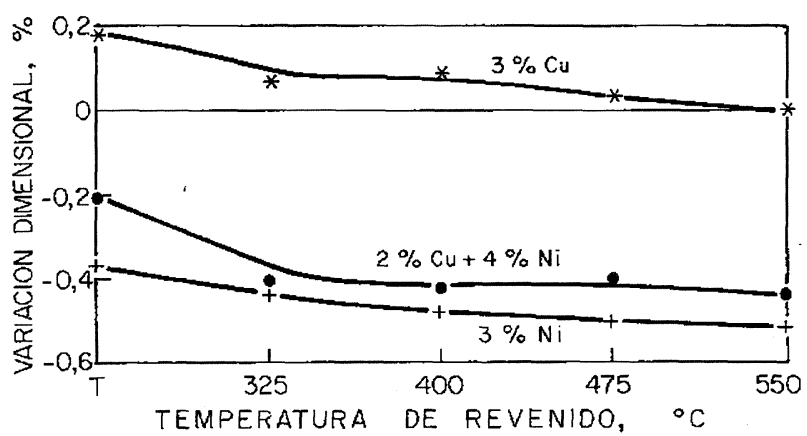

FIG. 2.- Valores de variación dimensional de los aceros $\mathrm{Fe}-1,5 \mathrm{Mo}-0,7 \mathrm{C}$, templados a $950{ }^{\circ} \mathrm{C}$ y revenidos a distintas temperaturas.

FIG. 2.-Dimensional change of $\mathrm{Fe}-0.7 \%$ C- $1.5 \%$ Mo steels, quenched at $950{ }^{\circ} \mathrm{C}$, and tempered at different temperatures.

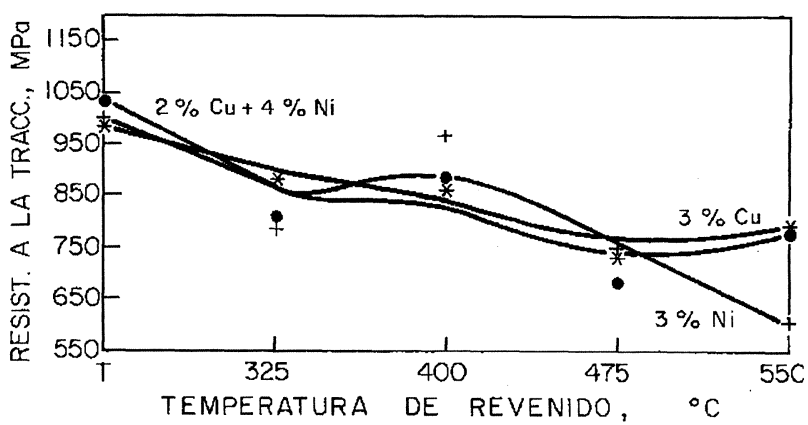

FIG. 3.- Valores de resistencia a la tracción de los aceros $\mathrm{Fe}-1,5-\mathrm{Mo}-0,7 \mathrm{C}$, templados a $950{ }^{\circ} \mathrm{C}$ y revenidos a distintas temperaturas.

FIG. 3.- Tensile strength of Fe-0.7\% C-1.5\% Mo steels, quenched at $950{ }^{\circ} \mathrm{C}$, and tempered at different temperatures.

\subsubsection{Alargamiento}

La figura 4 muestra los valores de alargamiento después del temple y revenido de todos los 


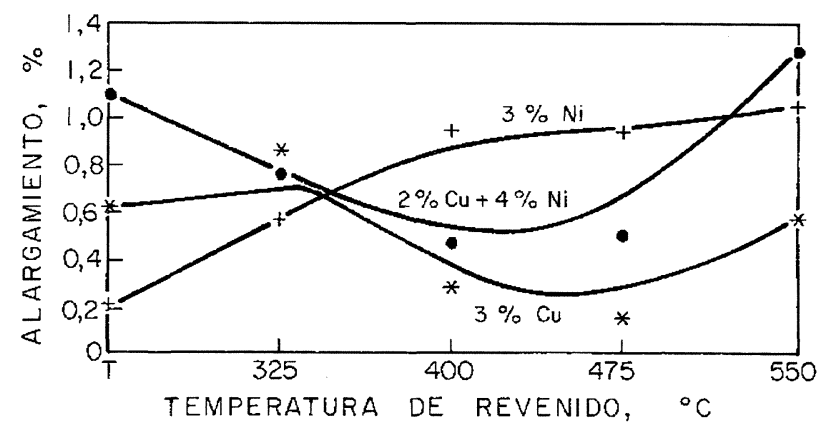

Fig. 4.- Valores de alargamiento de los aceros Fe$1,5 \mathrm{Mo}-0,7 \mathrm{C}$, templados a $950{ }^{\circ} \mathrm{C}$ y revenidos a distintas temperaturas.

FIG. 4.-Elongation of Fe-0.7\% C-1.5\% Mo steels, quenched at $950{ }^{\circ} \mathrm{C}$, and tempered at different temperatures.

materiales estudiados. Después del temple, las probetas con el $3 \% \mathrm{Cu}$ responden al revenido con una mejora del alargamiento $\mathrm{y}$, posteriormente, con un descenso del mismo; a partir de $475^{\circ} \mathrm{C}$, se observó un segundo aumento, alcanzando el valor del alargamiento casi el del estado de partida. El efecto que sobre el alargamiento tienen los tratamientos térmicos de la aleación con $3 \% \mathrm{Ni}$ es negativo, si lo comparamos con el estado sinterizado (1); no obstante, los posteriores revenidos se traducen en un aumento del mismo, brusco al inicio y más ligero a partir de $400{ }^{\circ} \mathrm{C}$. La aleación que contiene los dos elementos responde de manera análoga en lo que se refiere a las otras propiedades, es decir, se comporta, en general, de una forma intermedia entre el comportamiento de las aleaciones que contienen níquel o cobre.

\subsubsection{Dureza}

La figura 5 recoge los valores de dureza después del temple y revenido de todos los materiales estudiados. En los tres tipos de aleaciones ( $3 \% \mathrm{Ni}, 3 \%$ $\mathrm{Cu}$ y $2 \% \mathrm{Cu}+4 \% \mathrm{Ni}$ ), el efecto frente a la dureza es el mismo; es decir, como es de esperar, después del temple se produce un aumento de la dureza con respecto a la del estado sinterizado (1). A la temperatura de revenido de $400{ }^{\circ} \mathrm{C}$, se produce una ligera mejora con posterior descenso continuado de dureza.

\subsubsection{Metalografía}

La figura 6 muestra las microestructuras de los distintos materiales estudiados, templados y posteriormente revenidos.

En el Astaloy Mo con 0,7\% C - $3 \%$ Ni templado, se aprecian áreas austeníticas (que se correspon-

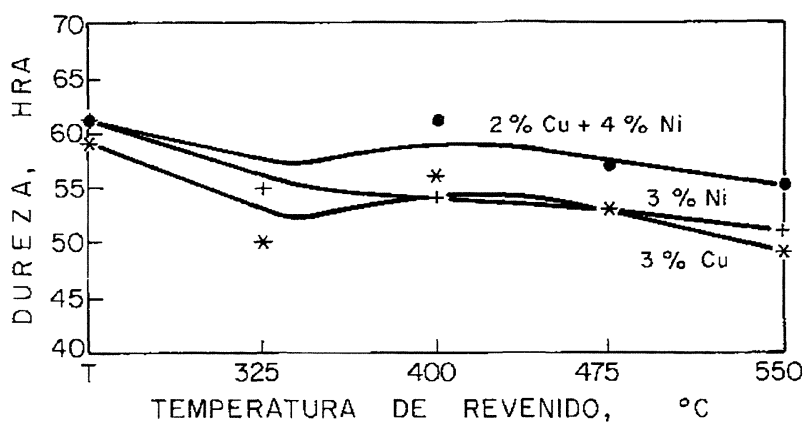

FIG. 5.- Valores de dureza de los aceros $\mathrm{Fe}-1,5$ Mo- $0,7 \mathrm{C}$, templados a $950{ }^{\circ} \mathrm{C}$ y revenidos a distintas temperaturas.

FIG. 5.- Hardness of Fe-0.7\% C-1.5\% Mo steels, quenched at $950{ }^{\circ} \mathrm{C}$, and tempered at different temperatures.

den con las que ya existían en el estado sinterizado) que, con el tratamiento de temple, no se han visto modificadas a causa de su alto contenido de níquel que las mantiene en forma metaestable. El resto del material, una vez austenizado, se ha transformado en martensita. En los distintos revenidos, la martensita se va descomponiendo progresivamente, siendo el efecto tanto mayor cuanto más elevada es la temperatura de revenido. Mediante el estudio realizado por determinación de microdureza (Fig. 7), se comprueba cómo los valores de ésta disminuyen en los microconstituyentes, desde los obtenidos en estado de temple (martensita) hasta los obtenidos en el revenido a $550{ }^{\circ} \mathrm{C}$; sin embargo, la austenita se endurece con la temperatura de revenido a causa de su posible transformación en bainita.

En el Astaloy Mo con 0,7 \% C - $3 \% \mathrm{Cu}$, templado y revenido a $325,400,475$ y $550{ }^{\circ} \mathrm{C}$, respectivamente, se observan, según se pasa del estado de temple a los distintos estados de revenido, los mismos fenómenos descritos para la aleación anterior, con níquel, a excepción de que ahora no hay presentes áreas de austenita metaestable. Al igual que en el caso de la aleación con níquel, al realizar la determinación de microdurezas de la martensita (Fig. 8), se observa que los valores van disminuyendo desde el estado de temple hasta los distintos estados de revenido.

En el Astaloy Mo con 0,7 \% C-2 \% Cu-4\% Ni templado y revenido a distintas temperaturas, se aprecian los mismos fenómenos descritos para los aceros al níquel tratados térmicamente, con respecto a la austenita residual. Pese a que en estos aceros se halla, además, cobre como elemento de aleación, a nivel microestructural se encuentran más las formaciones características que identifican la presencia de níquel, como, por ejemplo, la ya mencionada austenita metaestable y la porosidad por efecto Kirkendall (7), esta última muy generalizada. $\mathrm{Al}$ igual 

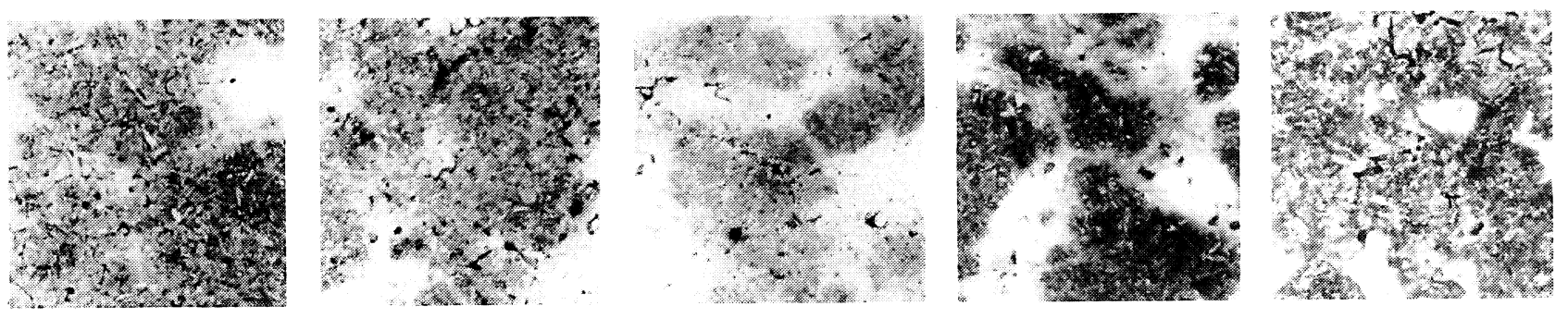

a)
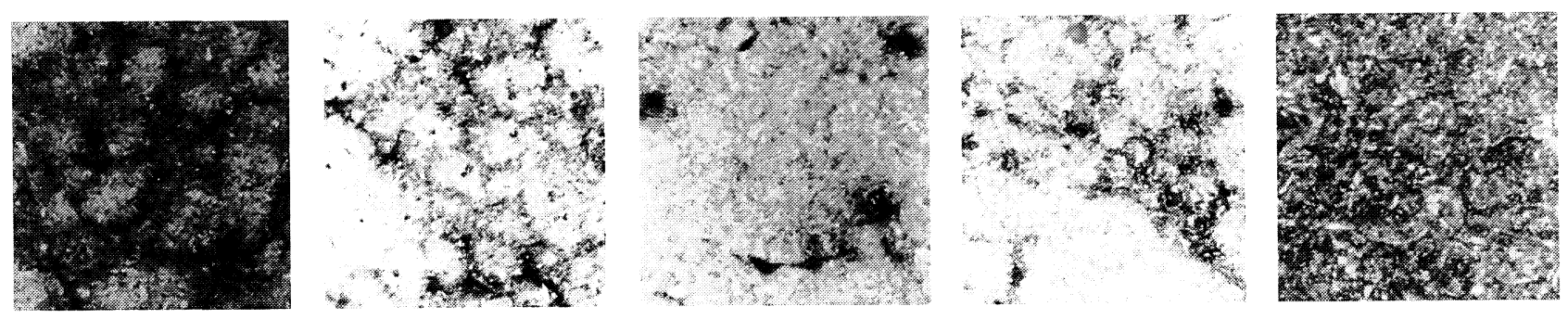

b)
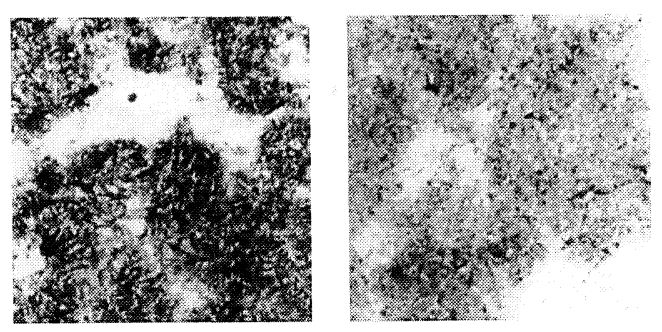

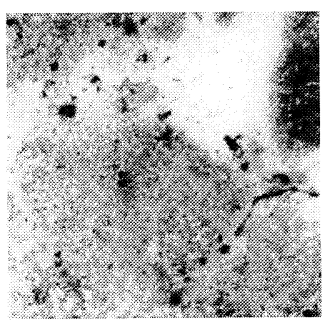

c)
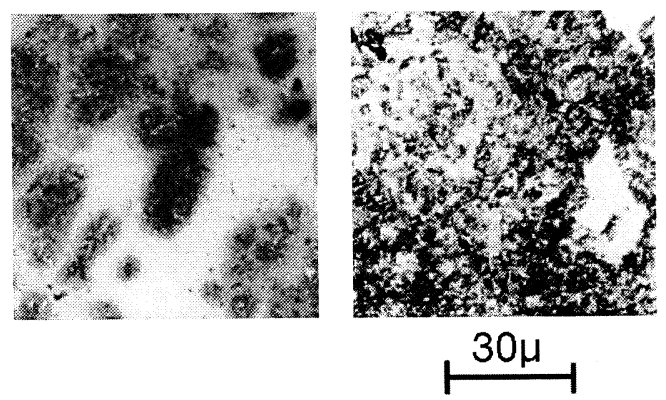

FIG. 6.- Microestructuras de los aceros Fe-0,7 C-1,5 Mo sinterizados a $1.120{ }^{\circ} \mathrm{C}$ durante 30 min, para distintos contenidos de cobre y de níquel.

a) Adición de $3 \% \mathrm{Ni}$.

b) Adición de $3 \% \mathrm{Cu}$.

c) Adición de $2 \% \mathrm{Cu}-4 \% \mathrm{Ni}$.

Ataque: Nital al $3 \%$.

FIG. 6.-Microstructures of Fe-0.7\% C-1.5\% Mo steels sintered at $1.120^{\circ} \mathrm{C}$ for 30 min, for different copper and nickel contents.

a) $3 \%$ Ni addition.

b) $3 \% \mathrm{Cu}$ addition.

c) $2 \% \mathrm{Cu}-4 \% \mathrm{Ni}$ addition.

$3 \%$ Nital etching.

que en los casos anteriores, la determinación de los valores de microdureza confirma cómo disminuyen dichos valores desde la martensita del estado de temple hasta los microconstituyentes del revenido a $550^{\circ} \mathrm{C}$. Al mismo tiempo, en las áreas de austenita va aumentando el valor de la microdureza desde el temple hasta el revenido a $550{ }^{\circ} \mathrm{C}$ (Fig. 9), donde se presenta una disminución progresiva.

\section{ANÁLISIS DE LOS RESULTADOS}

Los resultados obtenidos en las propiedades estudiadas son similares a los obtenidos por otros autores en condiciones parecidas de sinterizacióntratamiento (8 y 9 ).

El comportamiento dimensional y frente a los cambios de densidad de los materiales estudiados 


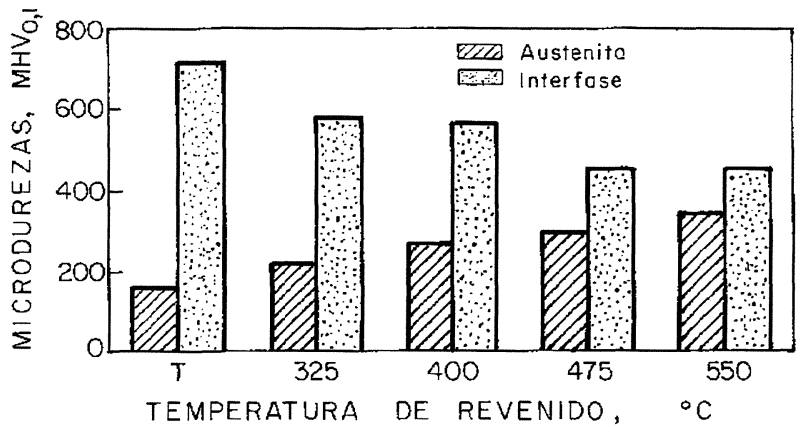

FIG. 7.- Valores de microdurezas de los microconstituyentes del Astaloy Mo con 0,7 \% C-3 \% Ni sinterizado a $1.120^{\circ} \mathrm{C}$ durante $30 \mathrm{~min}$, en estado de temple y para distintas temperaturas de revenido.

FIG. 7.- Microhardness of Astaloy Mo $0.7 \%$ $\mathrm{C}-3 \%$ Ni sintered at $1.120^{\circ} \mathrm{C}$ for $30 \mathrm{~min}$, quenched and tempered at different temperatures.

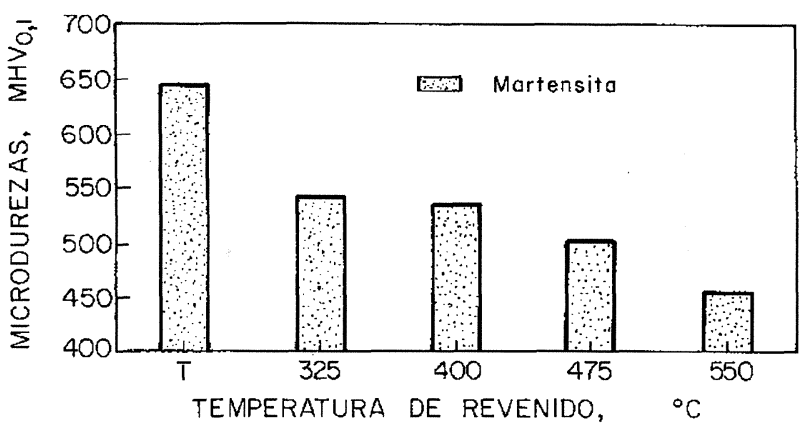

FIG. 8.- Valores de microdurezas de los microconstituyentes del Astaloy Mo con 0,7 \% C-3\% Cu sinterizado a $1.120^{\circ} \mathrm{C}$ durante $30 \mathrm{~min}$, en estado de temple y para distintas temperaturas de revenido.

FIG. 8.- Microhardness of Astaloy Mo $0.7 \%$ $\mathrm{C}-3 \% \mathrm{Cu}$ sintered at $1.120{ }^{\circ} \mathrm{C}$ for $30 \mathrm{~min}$, quenched and tempered at different temperatures.

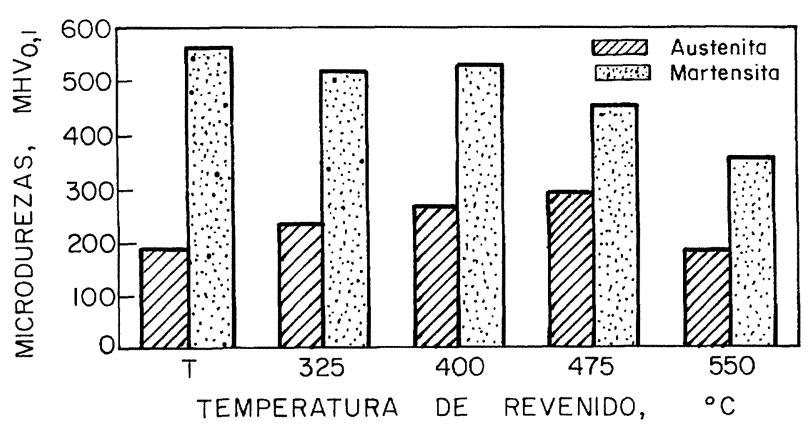

Fig. 9.- Valores de microdurezas de los microconstituyentes del Astaloy Mo con 0,7 \% C-2 \% $\mathrm{Cu}-4 \% \mathrm{Ni}$ sinterizado a $1.120{ }^{\circ} \mathrm{C}$ durante $30 \mathrm{~min}$ en estado de temple y para distintas temperaturas de revenido.

FIG. 9.- Microhardness of Astaloy Mo $0.7 \%$ C-2 $\% \mathrm{Cu}-4 \% \mathrm{Ni}$ sintered at $1.120{ }^{\circ} \mathrm{C}$ for $30 \mathrm{~min}$, quenched and tempered at different temperatures. tratados térmicamente es bueno en general, ya que estas propiedades no se ven sensiblemente afectadas por los mismos, sobre todo si se tienen en cuenta las variaciones previamente producidas a causa de la sinterización (1).

Con respecto a la resistencia a la tracción, el níquel provoca una disminución de la misma desde el estado de temple hasta el primer revenido; este hecho se explica porque la martensita transformada al templar, al pasar por sucesivos revenidos, se va transformando desde una estructura tetragonal a una estructura hexagonal compacta. Esta transformación va acompañada de un empobrecimiento de carbono de la matriz, con una disminución de la dureza y, por consiguiente, con un descenso de la resistencia a la tracción. A $400{ }^{\circ} \mathrm{C}$, la resistencia se eleva ligeramente, (según se deduce de la figura 3 ), aumento que puede explicarse porque la austenita residual se transforma en bainita inferior (lo cual se corrobora con el estudio de microdurezas de la figura 7), dando lugar a un aumento de dureza y resistencia cuando existe gran cantidad de austenita residual. A partir de $400{ }^{\circ} \mathrm{C}$, la resistencia disminuye debido al ablandamiento de la martensita primaria más la secundaria que posiblemente existe transformada a partir de la austenita. Con respecto a las aleaciones con cobre, en estas disminuye la resistencia según aumenta la temperatura de revenido. En este caso, sólo se transforma la martensita templada a martensita revenida con un ablandamiento, lo que produce una disminución de la resistencia. En cuanto a las aleaciones $\mathrm{Cu}-\mathrm{Ni}$, el comportamiento es el intermedio, ya que aunque el porcentaje de níquel es mayor que el de cobre, al difundir mejor este segundo elemento, su influencia es superior. Por estos efectos contrapuestos se reduce la cantidad de austenita que podría dar lugar al endurecimiento secundario o a la posible transformación de la austenita residual a martensita secundaria.

Se puede resaltar que en otro trabajo (10) se han obtenido resultados similares en los tratamientos de temple y revenido de Distaloys (del tipo $\mathrm{Fe}-1,5 \%$ $\mathrm{Cu}-4 \% \mathrm{Ni}-0,5 \% \mathrm{Mo}$ ), obteniendo un aumento de resistencia después del revenido a $300{ }^{\circ} \mathrm{C}$, propiciado por la transformación de la austenita retenida en bainita. En dicho trabajo, la temperatura a que se produce el aumento de resistencia es inferior, ya que el contenido de aleantes es menor $(0,5 \%$ Mo frente al 1,5\% Mo empleado en el presente trabajo).

\section{CONCLUSIONES}

En los aceros al Cu-Ni-Mo sinterizados estudiados, se mejoran sensiblemente las propiedades mecánicas después de tratamientos térmicos de temple y revenido, siendo la mejor aleación estudiada la de $2 \% \mathrm{Cu}-4 \% \mathrm{Ni}$. 
La mejor condición de tratamiento térmico entre los estudiados, desde el punto de vista de la resistencia a la tracción y de la dureza, es la de temple en aceite (austenización a $950{ }^{\circ} \mathrm{C} / 30 \mathrm{~min}$ ). Subsecuentes revenidos propician, en algunos casos, mejoras de la ductilidad.

La mejor combinación de tratamientos térmicos y composición en los materiales y condiciones estudiadas, desde el punto de vista de todas las propiedades mecánicas estudiadas, es: aleación con $3 \%$ $\mathrm{Ni}$, temple en aceite (desde $950{ }^{\circ} \mathrm{C}$ durante $30 \mathrm{~min}$ ) seguido de revenido a $400{ }^{\circ} \mathrm{C}$. Así, se obtienen unos valores de resistencia a la tracción de $966 \mathrm{MPa}$, alargamiento del $1 \%$ y dureza 54 HRA.

Después de los tratamientos térmicos de los aceros estudiados, las variaciones dimensionales no superaron en ningún caso el $0,5 \%$, siendo en los aceros aleados con $3 \% \mathrm{Cu}$ inferiores al 0,2\% (nulas en el caso del revenido a $550^{\circ} \mathrm{C}$ ).

\section{REFERENCIAS}

(1) Torralba, J.M., Candela, N., Ruiz Román, J.M., Cambronero, L.E.G. y Ruiz Prieto, J.M. Rev. Met. Madrid. 31 (1) 1994: 14-22.
(2) SANDEROW, H.I. High temperature sintering of ferrous $\mathrm{P} / \mathrm{M}$ components. Advances in Powder Metallurgy. Vol.1. Ed. Metal Powder Industries Federation, Princeton (NJ, EE.UU.) 1989: 187-202.

(3) LindQvist, B. A molybdenum-alloyed steel for surface hardening. Proc. World Congress on Powder Metall. Vol 2. Londres, 1990. 170-177.

(4) Furukimi, O., Maruta, K., Abe, T., Takajo, S. y Habu, K. Powder Metall. 34 (3), 1991: 212-214.

(5) Boulama, A., Brunel, G. y Ciceron, G. Processes involved during the elaboration of Fe-Mo alloys by powder metallurgy. Proc. Intern. Conf. on Powder Metall. PM'90. Vol. 2. Londres, 1990: 222-228.

(6) BocchinI, G.F. Recent advances in diffusion-bonded powders for high-strength PM applications. Workshop on Advanced Sintered Materials. Fundación Gómez Pardo, Madrid, Feb. 1994.

(7) LENEL, F.V. Powder Metallurgy: principles and applications. Ed. MPIF. Princeton, (NJ, EE.UU.) 1980: 269-284.

(8) Cambronero, L.E.G., Fernández, C., Torralba, J.M. y Ruiz Prieto, J.M. Powder Metall., 37 (1), 1994: 53-56.

(9) DAnninger, H. Powder Metall., 24 (2), 1992: 73.

(10) Torralba, J.M., RuIZ, J.M., LunA, J.M. y ZaPATA, W.C. Heat treatments of $\mathrm{Cu}-\mathrm{Ni}$-Mo sintered steels obtained from elemental mix and prealloyed powders. Proc. World Congress on Powder Metall. Vol. 2. Londres 2-6 Julio 1990: 69-74. 